\title{
Up-scalable ITO-free Organic Light Emitting Diodes based on Embedded Inkjet-Printed Copper Grids
}

Sergey M. Pozov ${ }^{1}$, Gerburg Schider ${ }^{2}$, Stefanie Voigt ${ }^{3}$, Franziska Ebert ${ }^{3}$, Karl Popovic $^{2}$, Felix Hermerschmidt ${ }^{4}$, Efthymios Georgiou ${ }^{1}$, Ignasi BurguésCeballos $^{1}$, Lukas Kinner ${ }^{2}$, Dieter Nees ${ }^{2}$, Barbara Stadlober ${ }^{2}$ Charlotte Rapley, $^{5}$, Richard Ward ${ }^{5}$, Stelios A. Choulis ${ }^{1}$, Emil J. W. List-Kratochvil ${ }^{4,6}$ and Christine Boeffe $1^{3 *}$

\footnotetext{
${ }^{1}$ Molecular Electronics and Photonics Research Unit, Department of Mechanical Engineering and Materials Science and Engineering, Cyprus University of Technology, 45 Kitiou Kyprianou Street, 3041 Limassol, Cyprus

${ }^{2}$ Materials-Institute for Surface Technologies and Photonics, Joanneum Research Forschungsgesellschaft mbH, Franz-Pichler-Straße 30, A-8160 Weiz, Austria

${ }^{3}$ Fraunhofer Institute for Applied Polymer Research, Geiselbergstraße 69, 14476 Potsdam-Golm, Germany

${ }^{4}$ Institut für Physik, Institut für Chemie \& IRIS Adlershof, Humboldt-Universität zu Berlin, BrookTaylor-Straße 6, 12489 Berlin, Germany

${ }^{5}$ Intrinsiq Materials Ltd, Building Y25, Cody Technology Park, Ively Road, Farnborough, Hampshire GU14 OLX, United Kingdom

CR: current address: Chemistry Department, Imperial College

${ }^{6}$ Helmholtz-Zentrum Berlin für Materialien und Energie GmbH, Brook-Taylor-Straße 6, 12489 Berlin, Germany
}

*corresponding authors: christine.boeffel@iap.fraunhofer.de, stelios.choulis@cut.ac.cy 


\begin{abstract}
We report on ITO-free OLEDS with a transparent hybrid $\mathrm{Cu}$ nanoparticle grid/PEDOT:PSS electrode processed in ambient conditions. An experimentally based methodology was implemented, where studies on alternative PEDOT:PSS derivatives and $\mathrm{Cu}$ grid design were performed, to gradually increase the efficiency of lab scale ITO-free OLEDs. To further increase electrode performance, inkjet-printed (IJP) Cu-grids are embedded to flatten the electrode, reduce leakage current and enhance homogeneity and efficiency. Finally, embedded $\mathrm{Cu}$ based ITO-free OLEDs showed current and power efficiencies comparable to reference ITO-based OLEDs. Methods to manufacture large area flat embedded IJP Cu-electrodes on glass and flexible substrates are presented and upscaling prospects of the proposed ITO-free electrode are discussed.
\end{abstract}




\section{Introduction}

Organic semiconductors have raised significant interest in research and industry over the last three decades. Despite the prominence of conventional silicon-based semiconductor industry, several advantages such as cost effectiveness, light weight and large area processability promise a wide range of commercial applications for organic semiconductors. Key benefits such as the usability of flexible substrates and the bright colors of organic light emitting diodes (OLEDs) can lead to competitive solutions as TV screens or profitable displays for mobile phones. Most types of commercially available OLEDs still use indium tin oxide (ITO) as the transparent electrode, but future manufacturing and application as roll to roll fabricated devices are hindered by the brittleness of ITO and the limited resources of indium ${ }^{1}$. Therefore, many efforts are being made to effectively replace ITO and reduce the overall cost of organic optoelectronic devices. Alternatives to ITO electrodes as applied in organic optoelectronics are usually classified into five different groups of materials: i) highly conductive polymers ${ }^{2-}$ ${ }^{4}$, ii) metal oxides ${ }^{5}$, iii) carbon based conductors ${ }^{6}$, iv) metallic nanomaterials ${ }^{7-}$ ${ }^{9}$, and v) hybrid electrodes ${ }^{10,11}$. Among all these solutions, inkjet-printed metal nanoparticle grids usually combined with conductive polymer PEDOT:PSS have shown the best perspectives in efficient and simple replacement of ITO, in both organic photovoltaic (OPV) ${ }^{12-17}$ and organic light emitting diode (OLED) devices ${ }^{18-20}$.

Almost all the reported work of inkjet-printed metal grids used silver nanoparticle inks because of its high conductivity and corrosion resistance. However, the high cost and rareness of silver metal have led to the investigation of less expensive material, which will further decrease the cost of ITO-free optoelectronic devices. Copper is the $26^{\text {th }}$ most abundant element in the Earth's crust with $0.0068 \%$ availability and therefore it is cheaper than silver ${ }^{21}$. The main drawback of copper is that it is thermodynamically unstable at atmospheric condition and is easily oxidized into either $\mathrm{Cu}_{2} \mathrm{O}$ or $\mathrm{CuO}$, both of which are less conductive compared to copper. To date the applications of inkjet-printed Cu NP inks are limited, mainly to patterned conductive paths on glass ${ }^{22-24}$ and flexible ${ }^{25,26}$ substrates. In 2015, Norita et al. were the first to successfully use inkjet-printed $\mathrm{Cu}$ NP electrodes in organic thin-film transistors ${ }^{27}$. In 2016, Polino et al. investigated a set of three 
commercial $\mathrm{Cu}$ nano-inks in order to benchmark their potential to form conducting printed structures for future applications in organic electronic devices ${ }^{28}$. It was concluded that Cu NP grid corroded by the acidic nature of PEDOT:PSS hole injection layer applied on-top of the grid, and that further investigation was necessary to achieve a functional $\mathrm{Cu}$ NP electrode for optoelectronic applications. In 2018, Georgiou et al. successfully fabricated the first ITO-free non-embedded $\mathrm{Cu}$ grid based organic photovoltaics with 3.4\% efficiency where the reference ITO-based device was $4.9 \%{ }^{16}$.

In this work we present for the first-time efficient ITO-free OLEDs comprising IJP Cu nanoparticle grid/PEDOT:PSS as bottom hybrid electrode. We present a detailed study on the influence of PEDOT:PSS derivatives on the performance of Cu-grid based electrode and we investigate the effect of the reverse nanoimprinting transfer of printed grids and grid design on the performance of the ITO-free OLEDs. We demonstrate that the embedding process can be used to planarize the proposed Cu-based electrodes on flexible and glass substrates and we discuss the up-scaling approaches towards large area ITO-free OLEDs that are based on embedded inkjet-printed copper grids.

\section{Results and Discussion}

Both the printing and sintering of $\mathrm{Cu}$ grids were performed in ambient conditions, using a developed $\mathrm{Cu}$ nano-ink produced using copper nanoparticles of approximately $100 \mathrm{~nm}$ in diameter, formed by a high temperature plasma synthesis method (CI-005, synthesized by Intrinsiq Materials). The good printability of CI-005 Cu nano-ink formulation and constant wavelength (CW) laser sintering have already been demonstrated in our previous work ${ }^{16}$, therefore the same conditions were adapted. Even though the best conductivities are achieved using reduced atmosphere thermal sintering which can result in $\mathrm{Cu}$ film conductivities from $20 \%$ up to $30 \%$ of $\mathrm{Cu}$ bulk conductivity ${ }^{29}$. On other hand photonic and laser sintering have shown a great potential over the past years ${ }^{30-33}$. The high conductivities achieved with photonic/laser sintering together with their advantages of extremely short processing times, high spatial precision and applicability under ambient conditions, makes these sintering methods 
alternatives to thermal sintering, which usually requires long duration of heating and controlled atmosphere.

The mechanism of laser sintering relies on the absorption of the emitted photonic energy by the printed nanoparticles resulting in the removal of the nanoparticle capping agent and the coalescence of the particles. Figure 1a and b show SEM images of the surface of $\mathrm{Cu}$ NP layer before and after sintering, respectively. The effect of laser sintering can be observed in the necking and merging of Cu NPs promoted by the generated thermal energy resulting from absorption of radiant energy, and therefore providing high electrical conductivity of $\sim 36000 \mathrm{~S} / \mathrm{cm}$.
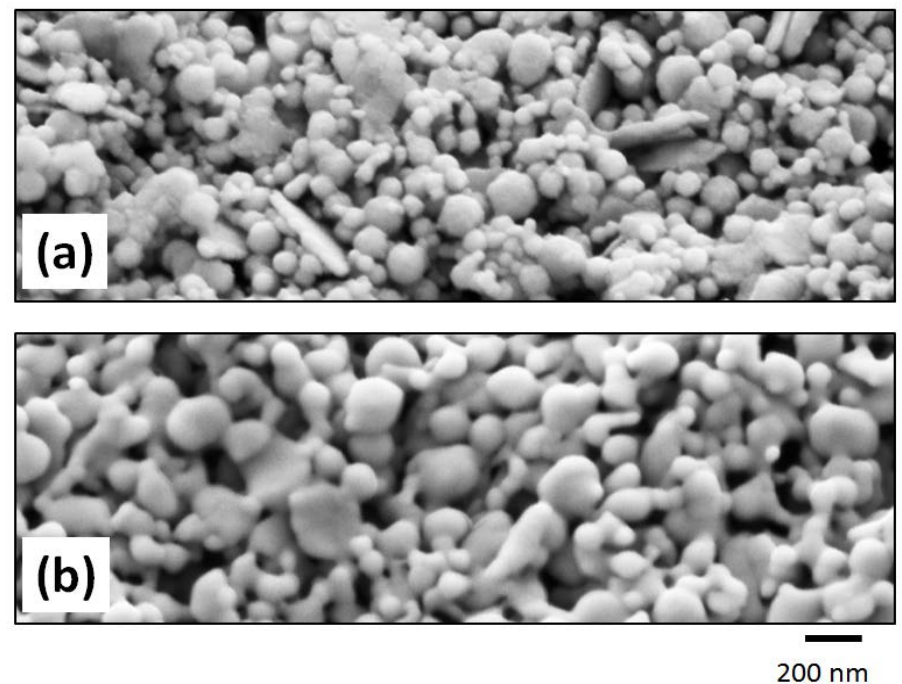

Figure 1: SEM micrographs of inkjet-printed Cu-ink (a) before and (b) after laser sintering.

\subsection{Optimization of lab-scale ITO-free OLEDs on glass substrates and their implementation on flexible substrates}

\subsubsection{Effect of PEDOT: PSS conductivity on hybrid electrode}

According to our previous work on ITO-free OLEDs using Ag NP grids ${ }^{18}$, electrodes consisting of 8 parallel line grids were concluded to be the most efficient geometry. Therefore, our initial studies were performed using 8 parallel line $\mathrm{Cu}$ NP grids covered with various PEDOT:PSS formulations, in order to define the best combination for hybrid electrodes. The effect of the conductivity of PEDOT:PSS on the performance of ITO-free OLEDs was examined using three different PEDOT:PSS formulations with different conductivities, as measured using the four-point probe method. Low conductive PEDOT:PSS 
Clevios PVP AI4083 ( 0.001 S/cm) was initially selected since it is the same formulation that was used in ITO-based reference devices. A second PEDOT:PSS, FCE with 4 orders of magnitude higher electrical conductivity than AI4083 ( 10 S/cm) was also investigated. As has been reported previously, FCE formulation has an additional ability to planarize the inkjet-printed grid surface 19,34. Finally, PEDOT:PSS PH500 formulation was selected to test even higher conductivity $(\sim 500 \mathrm{~S} / \mathrm{cm})$. All the organic layers were spin coated, while $\mathrm{LiF} / \mathrm{Al}$ cathode was thermally evaporated to fabricate ITO-free (8-line $\mathrm{Cu} \mathrm{NP}$ grid/PEDOT:PSS/Super Yellow/LiF/Al) OLED devices and compare them with ITO-based reference devices used throughout this work (ITO/PEDOT:PSS (AI4083)/Super Yellow/LiF/Al). Detailed experimental conditions are presented in the respective section of this work.

Figure 2a represents the current efficiency (CE) characteristics of the examined OLED devices. A clear trend in device performance was observed, where CE increased when using a PEDOT:PSS formulation with higher conductivity. The best result was achieved with the most conductive PEDOT:PSS PH500 formulation, that resulted in a maximum CE of $2.5 \mathrm{~cd} / \mathrm{A}$, when the reference was $5.0 \mathrm{~cd} / \mathrm{A}$. Similarly, luminance characteristics as shown in Figure 2c revealed that PH500 formulation works much better than AI4083 and FCE, in terms of maximum luminance and light emission homogeneity. Semi-log current density - voltage (JV) characteristics of the devices shown in Figure 2d revealed that all ITO-free OLEDs suffered from high leakage current in comparison to the reference device. The PEDOT:PSS PH500 based ITO-free OLEDs had the highest leakage current. 


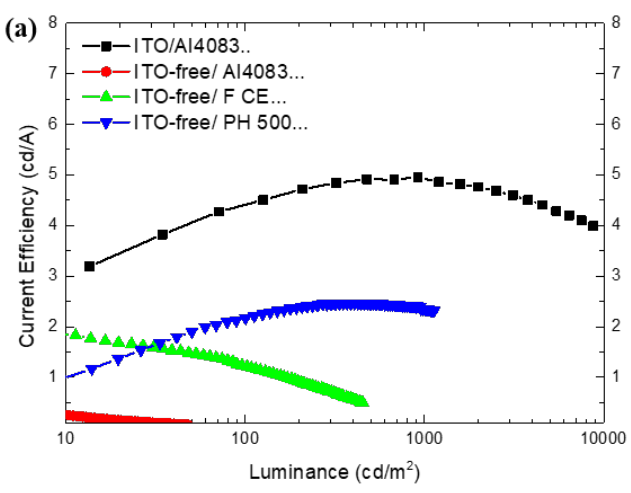

(c)
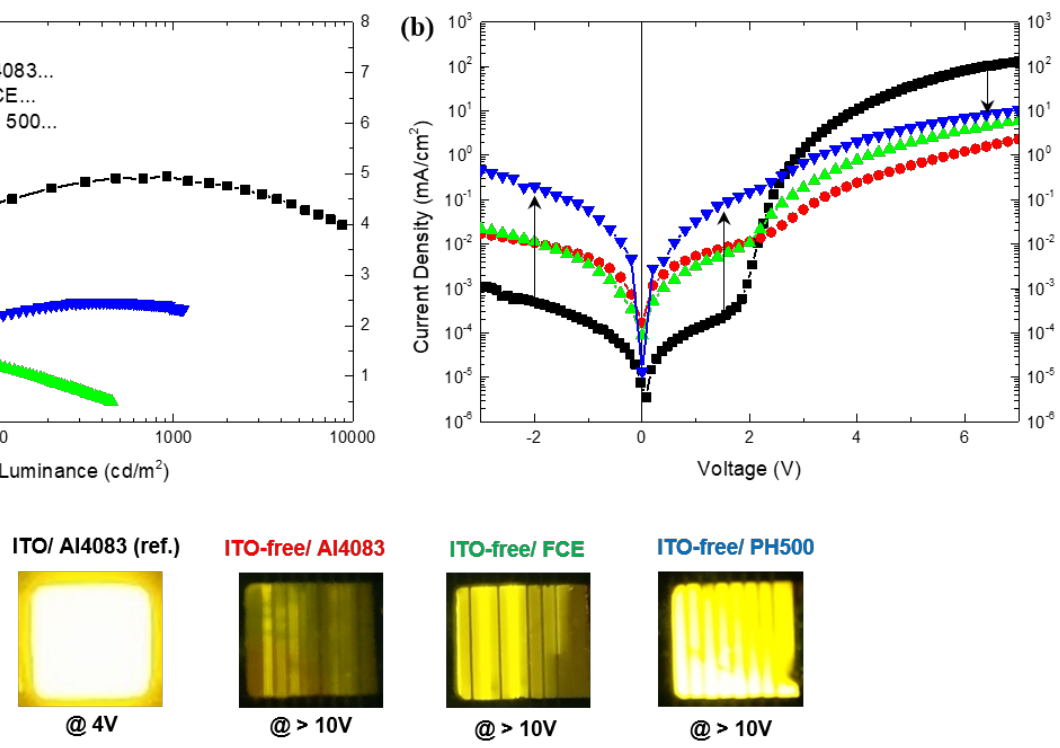

Figure 2: EL performance of ITO-free OLED devices using PEDOT:PSS formulations with different conductivities spin coated on 8 parallel line Cu NP grid, (a) Current efficiency characteristics, (b) semi-log JV characteristics and (c) photographs of $9 \mathrm{~mm}^{2}$ OLED devices under forward bias.

As it had been suggested before, the concentration of PSS in bulk PEDOT:PSS is crucial for its electron blocking properties ${ }^{35}$, therefore the lower is the PSS the worse is the electron blocking properties of PEDOT:PSS. Therefore, one of the reasons of increased leakage current of PH500 based devices in comparison with the other PEDOT:PSS formulations is attributed to its lower PSS content ${ }^{36}$.

Another important factor in preventing leakage current, is to address the homogeneity of individual layers of the OLED device stack. A typical problem in inkjet-printed metallic grids lies in their limited geometrical characteristics and specifically their heights, which are usually much thicker than all the organic films of the device. In this work, the measured height of $\mathrm{Cu}$ grid lines was approximately $405 \pm 51 \mathrm{~nm}$, as shown in Figure 3a. To avoid short-circuit paths, grids were overcoated using thick $(>100 \mathrm{~nm})$ PEDOT:PSS layers. Although the overcoating of $\mathrm{Cu}$ grid was achieved, profilometric scans revealed that planarization of the lines was not successful, and 'wavy' like interfaces were produced, creating low resistance paths between the two electrodes, as shown in the schematic illustration of Figure 3b. The generation of low resistance paths introduced short circuit and promoted leakage current, thereby reducing device performance. Therefore, it was concluded that leakage current was a combination 
of leakage currents attributed to intrinsic properties of highly conductive PEDOT:PSS PH500 and geometrical characteristics of IJP Cu grid.

(a)

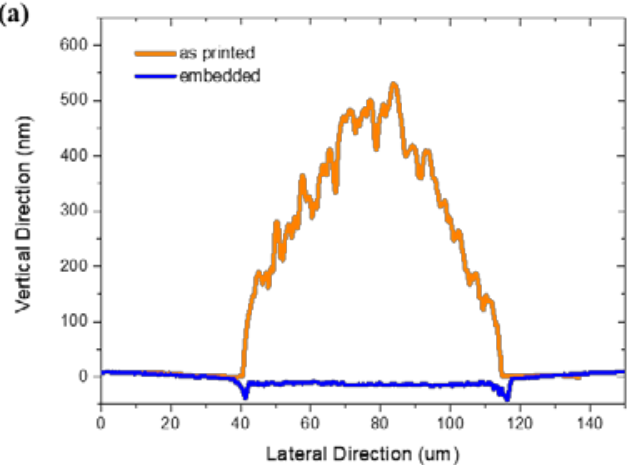

(c)

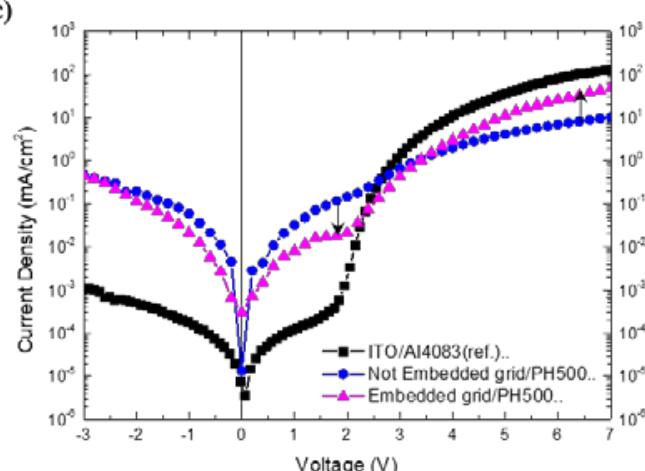

(b)

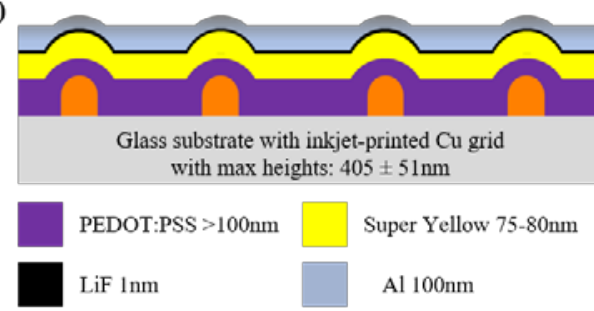

(d)

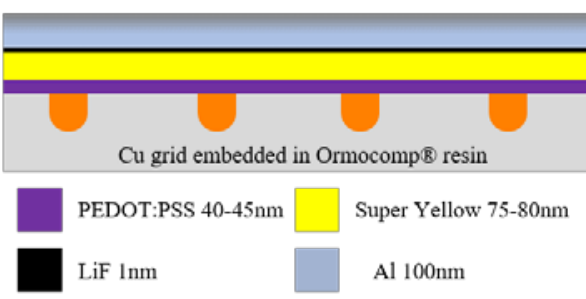

Figure 3: Comparison of embedded and not embedded Cu grids (a) Cross section profiles of inkjet-printed, dried and sintered Cu NP before and after embedding, (b) schematic illustration of ITO-free OLED device on-top of not embedded Cu grid with overcoated PEDOT:PSS layer, (c) semi-log JV characteristics of OLED devices fabricated (d) schematic illustration of ITO-free OLED device on-top of embedded Cu grid

\subsubsection{Grid flattening using reverse nanoimprinting transfer}

One possible solution to reduce leakage current is the increasing PSS content, however this will negatively affect both the conductivity of PH500 formulation 37 and performance of ITO-free OLED devices. A better solution would be the optimization of geometrical characteristics of the printed grid. Planarizing the surface of the grid, by reverse nanoimprinting transfer method which was initially proposed by Burgués-Ceballos et al. in $2014^{15}$, ensures flat surface and reduced leakage currents, and it has been already used in literature ${ }^{16,19}$. As shown in Figure 3a, printed $\mathrm{Cu}$ NP line profile is totally flattened after embedding, while its electrical and optical properties remained unaffected by the whole process. Based on the flattened electrode surface, it was assumed that the interfaces of 
device layers were planarized as shown in the schematic illustration of Figure 3d. Semi-log JV characteristics of Figure 3c revealed important reduction in leakage current of embedded $\mathrm{Cu}$ grid in comparison to not embedded grid. Leakage current reduced almost by one order of magnitude and current injection at higher voltages improved, approaching reference behavior. After the turn on voltage ( 2.6V) at the same voltage more current was injected in embedded devices than in the not-embedded.

The improvement in charge injection was also confirmed by photocurrent measurements, measuring the generated photocurrent (PCT) in complete OLED devices ${ }^{37}$. Figure 4 a presents the PCT maps of ITO-based reference and ITO-free OLEDs with embedded and not embedded Cu grid. In both cases the high-quality PCT maps revealed the printed $\mathrm{Cu}$ lines, the exact device area and the generated photocurrent. The overall photocurrent generation and distribution in embedded devices is more homogeneous across the whole device area than for the not embedded grid devices and have a trend towards the reference devices. It was also observed that not-embedded grids suffered from disconnected conductive paths originating mainly from the fabrication process, leading to lower current injection and inhomogeneous distribution.

Upon investigating the luminance characteristics (Figure 4b), significant improvements were achieved by embedding the $\mathrm{Cu}$ grid. Turn on voltage was almost identical to reference devices, indicating efficient charge injection, and maximum luminance of approximately $10,000 \mathrm{~cd} / \mathrm{m}^{2}$ with homogeneous light emission were achieved. Improvement of charge injection and reduction of leakage current lead to increase in the overall CE of the embedded ITO-free OLEDs as shown in Figure 4c. Maximum CE of embedded devices increased up to $3.0 \mathrm{~cd} / \mathrm{A}$ in comparison to $2.5 \mathrm{~cd} / \mathrm{A}$ of not-embedded devices, indicating a $20 \%$ improvement. 

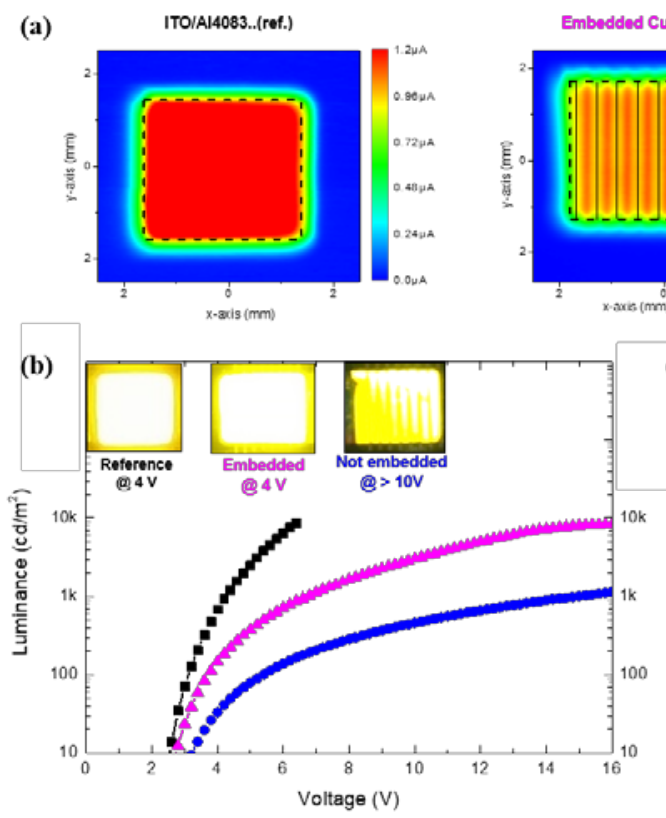
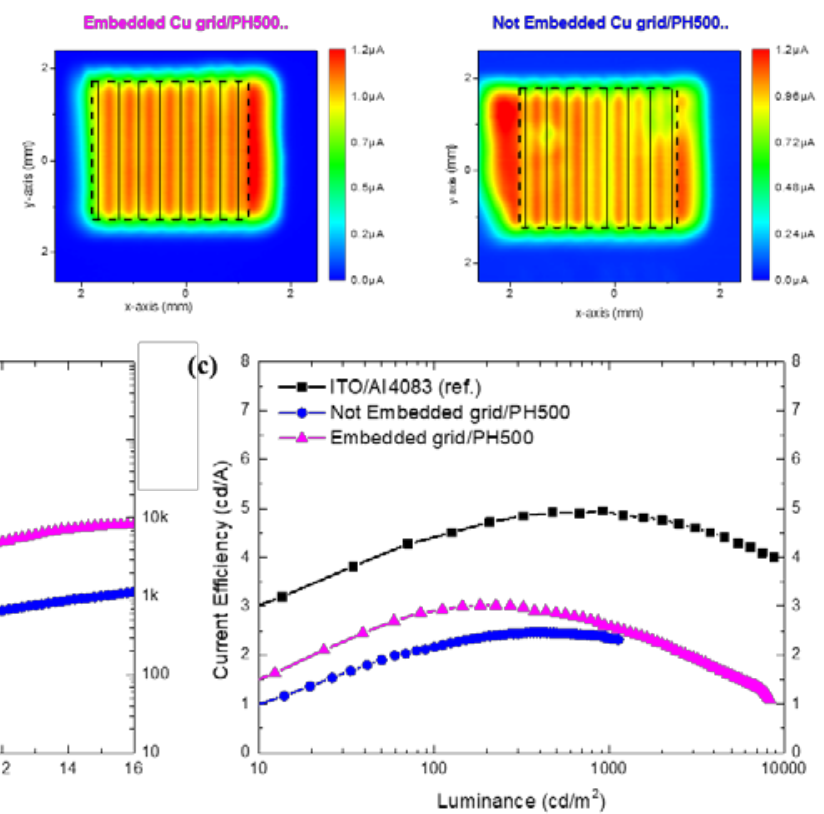

Figure 4: Characterization of ITO-free $9 \mathrm{~mm}^{2}$ OLED devices based on embedded and not embedded 8 parallel line Cu grid (a) photocurrent maps - due to the large scanning area, the effects of busbar are also presented in ITO-free devices (b) luminance characteristics, inset images show photographs of investigated devices under operation (c) current efficiency as a function of operating luminance.

\subsubsection{Embedded grid layout optimization}

Since device performance is strongly dependent on the electrical properties of the hybrid electrode, it was decided to further optimize the grid layout in terms of its geometrical characteristics in order to provide the best combination of grid layout and conductivity. Based on our investigations using different numbers of parallel lines as shown in Table S1 Supporting Information, the optimum grid coverage area was determined to be $23.5 \%$ which translates to 8 parallel lines grid layout.

Subsequently, the advantages of a honeycomb layout were investigated, which could provide a better balance between lowest possible coverage and sufficiently and homogeneously distributed number of conductive paths, compared to other geometries such as triangular or rectangular geometry or parallel lines. IJP Ag NP honeycomb grid structures have been successfully used in ITO-free OPVs 13,17 and ITO-free OLEDs ${ }^{19}$. Therefore, ITO-free OLEDs with embedded honeycomb grid layout were inkjet-printed with a geometrical height (distance from opposite edges of hexagon) of $\mathrm{H}=0.75 \mathrm{~mm}$ to achieve the optimized grid covered area of 
23.5\%. Honeycomb based devices were compared with same grid coverage area, embedded 8 parallel line ITO-free OLEDs in order to define its advantages.

JV characteristics in Figure 5a revealed that honeycomb-based devices had more current flow at the same voltage in comparison to parallel line grid. In addition, this significant improvement in electrical properties of honeycomb-based electrodes was confirmed by a better device performance, as can be seen in Figure 5b. The maximum CE increased from $3.0 \mathrm{~cd} / \mathrm{A}$ to $3.6 \mathrm{~cd} / \mathrm{A}$ when substituting the optimized parallel line grid with a honeycomb grid layout, consequently resulting in an extra 20\% improvement. Replacing the 8-parallel line grid by a honeycomb grid improved the electrical properties of the hybrid electrode. As it can be observed in PCT maps of complete ITO-free OLED devices Figure 5c, higher photocurrent values and more homogeneous current distribution along the whole device area indicated the important difference between the honeycomb and parallel line grid.

(a)

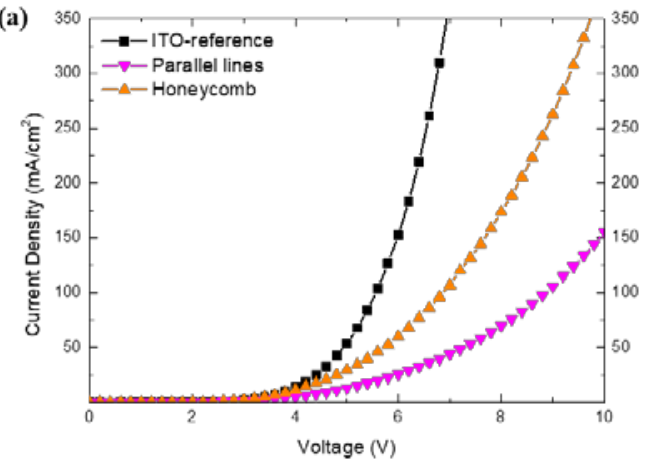

(c)

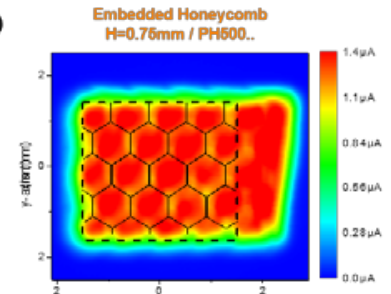

$x \operatorname{six}(\operatorname{mm})$

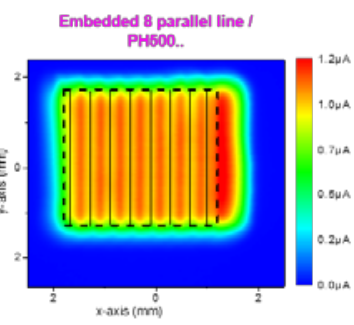

(b)

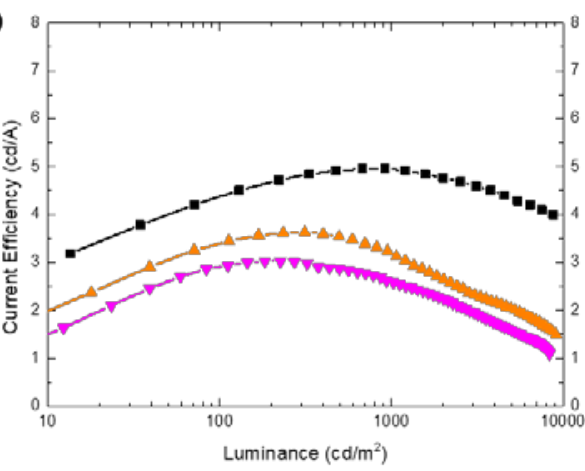

(d)

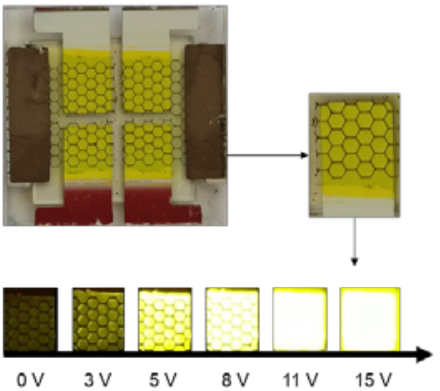

Figure 5: Honeycomb vs parallel line grid layout, both with $23.5 \%$ grid covered area, (a) linear JV characteristics, (b) current efficiency (c) photocurrent maps of ITO-free $9 \mathbf{~ m m}^{2}$ OLED devices, and (d) photograph of the sample comprised with four optimized lab-scale 
9mm² ITO-free OLED devices using embedded honeycomb Cu grid. Photographs of device luminance were taken at the voltages shown.

To get a clear view on the electroluminescent (EL) performance of the final and optimized ITO-free OLEDs as shown in Figure 5d, statistical analysis was performed using 15 different devices. In this analysis another reference device (reference 2) was implemented, using the following structure ITO/ PH500 as the bottom electrode. The fabrication of the reference 2 was useful to compare ITObased and Cu grid ITO-free devices, using the same PEDOT:PSS formulation. Therefore the only difference was the substitution of presence or not of ITO. By analyzing the data in Table 1, deviations in maximum current efficiency and maximum power efficiency of optimized ITO-free $\mathrm{Cu}$ grid/PH500 against ITO/PH500 were $16.6 \%$ and $20.6 \%$ respectively, which is much lower than the deviation with our standard reference 1 (ITO/PEDOT:PSS AI4083) 28\% in CE and $24.4 \%$ in PE. Georgiou et al. ${ }^{16}$ investigated the compatibility of $\mathrm{Cu}$ grids with PEDOT:PSS and the effect of thermal annealing on Cu grid conductivity. It was concluded that $\mathrm{Cu}$ grid is compatible with PEDOT:PSS, but its conductivity is very sensitive to temperature exposure under ambient conditions. Therefore, based on the fact that all PEDOT:PSS films fabricated in this work, were annealed at $140^{\circ} \mathrm{C}$ for 20 minutes in ambient conditions, and that OLED devices require high electrode conductivities, Cu grid conductivity was reduced and therefore the EL performance of ITO-free devices was deviated from ITO-based reference values.

As a final step in our investigation on lab-scale ITO-free OLEDs, the importance of grid-design in ITO-free OLED devices was underlined by fabricating devices that comprised of PH500 only layer, as the bottom electrode, namely the device structure was $\mathrm{PH} 500 / \mathrm{SY} / \mathrm{LiF} / \mathrm{Al}$ and the devices were compared with the optimized ITO-free OLEDs as shown in Figure S 1, Supporting information. The choice of honeycomb metal grid improves significantly the electrical properties of the ITO-free bottom electrode, promoting more effective charge injection and increasing the maximum luminance and therefore the overall performance of ITO-free OLEDs. 
Table 1: Statistical analysis of electroluminescent performance of optimized Cu NP ITOfree OLED, ITO/AI4083 (reference 1) and ITO/PH 500 (reference 2) using statistic sample size of 15 different devices. Average values are presented, together with standard deviation, while maximum values are presented in brackets.

\begin{tabular}{|c|c|c|c|c|}
\hline Device structure & $\begin{array}{c}\text { Turn on voltage } \\
\text { (at } 10 \mathrm{~cd} / \mathrm{m}^{2} \text { ) } \\
\text { [V] }\end{array}$ & $\begin{array}{c}\text { Max. } \\
\text { Luminance } \\
{\left[\mathbf{c d} / \mathbf{m}^{2}\right]}\end{array}$ & $\begin{array}{l}\text { Max. Current } \\
\text { efficiency } \\
\text { [cd/A] }\end{array}$ & $\begin{array}{c}\text { Max. Power } \\
\text { Efficiency } \\
{[\mathrm{lm} / \mathrm{W}]}\end{array}$ \\
\hline Reference 1 & 2.6 & $\sim 10 \mathrm{k}$ & $4.7 \pm 0.2(5.0)$ & $4.0 \pm 0.5(4.5)$ \\
\hline Reference 2 & 2.6 & $\sim 10 \mathrm{k}$ & $3.8 \pm 0.4(4.2)$ & $3.0 \pm 0.7(4.1)$ \\
\hline ITO-free; $0.75 \mathrm{~mm}$ & 2.6 & $\sim 10 \mathrm{k}$ & $3.3 \pm 0.6(3.6)$ & $2.7 \pm 0.6(3.4)$ \\
\hline \multicolumn{5}{|c|}{ Reference 1: ITO/AI4083/SY/LiF/Al } \\
\hline \multicolumn{5}{|c|}{ Reference 2: ITO/PH 500/ SY/LiF/Al } \\
\hline
\end{tabular}

\subsubsection{Optimized ITO-free OLED devices using flexible substrates}

As a next step, fabrication of lab-scale ITO-free OLEDs on flexible substrate was investigated. Starting with Cu NP grid printing and sintering, several challenges were presented. Even though printability could be controlled, direct laser sintering of $\mathrm{Cu}$ NP grid on flexible substrate induced high temperatures that melted and destroyed the flexible substrate. Therefore, $\mathrm{Cu}$ NP grids were printed and sintered on a sacrificial glass substrate and then transferred on a flexible target foil (Melinex 506) using the reverse nanoimprinting method and NILcure JR82 as the transfer resist (proprietary development of Joanneum Research). The fabricated flexible ITO-free OLEDs devices shown in Figure S2 offered great potential and perspectives for future investigations. 


\section{Up-scaling Prospects of the $\mathrm{Cu}$ based Printed Electrode}

Perspectives of $\mathrm{Cu}$ NP grid upscaling were also investigated in this work to pave the way for large area applications, e.g., in the field of lighting systems. For the printing of larger area grids, the only parameter that needed to be changed was the substrate temperature. Higher temperature was used in order to promote faster drying and avoid ink spreading due to the longer duration of printing process. As shown in Figure 6, Cu NP grids were successfully printed on $1.5 \times 1.5 \mathrm{~cm}^{2}, 5 \times 5$ $\mathrm{cm}^{2}$ and $15 \times 15 \mathrm{~cm}^{2}$ glass substrates. All large area $\mathrm{Cu}$ NP grids were printed on Hellmanex ${ }^{\circledR}$-cleaned Corning ${ }^{\circledR}$ Eagle XG ${ }^{\circledR}$ glass substrates to avoid the need for any further treatment. This is of high importance because printing of large area patterns can require considerable printing time. Consequently, semi-stable surface treatments such as oxygen plasma can suffer from a gradual modification of the surface properties during the printing process with a high risk for modification of the printed pattern. Additionally, grids were deliberately printed with different patterns in order to show the freedom of design of the inkjet printing technique. Printing times depend generally on the printing pattern and the employed parameters, where honeycomb grids required typically more printing time compared to line grids with similar grid coverage. Therefore, when investigating the printing properties for the $15 \times 15 \mathrm{~cm}^{2}$ substrates, significant differences were observed, as 3x3 large area $\mathrm{Cu}$ line grids could be printed within 30 minutes with optimized printing parameters, while $3 \times 3$ honeycomb patterns required more than 60 minutes printing time, durations that also dependent on how many nozzles were used for jetting. 


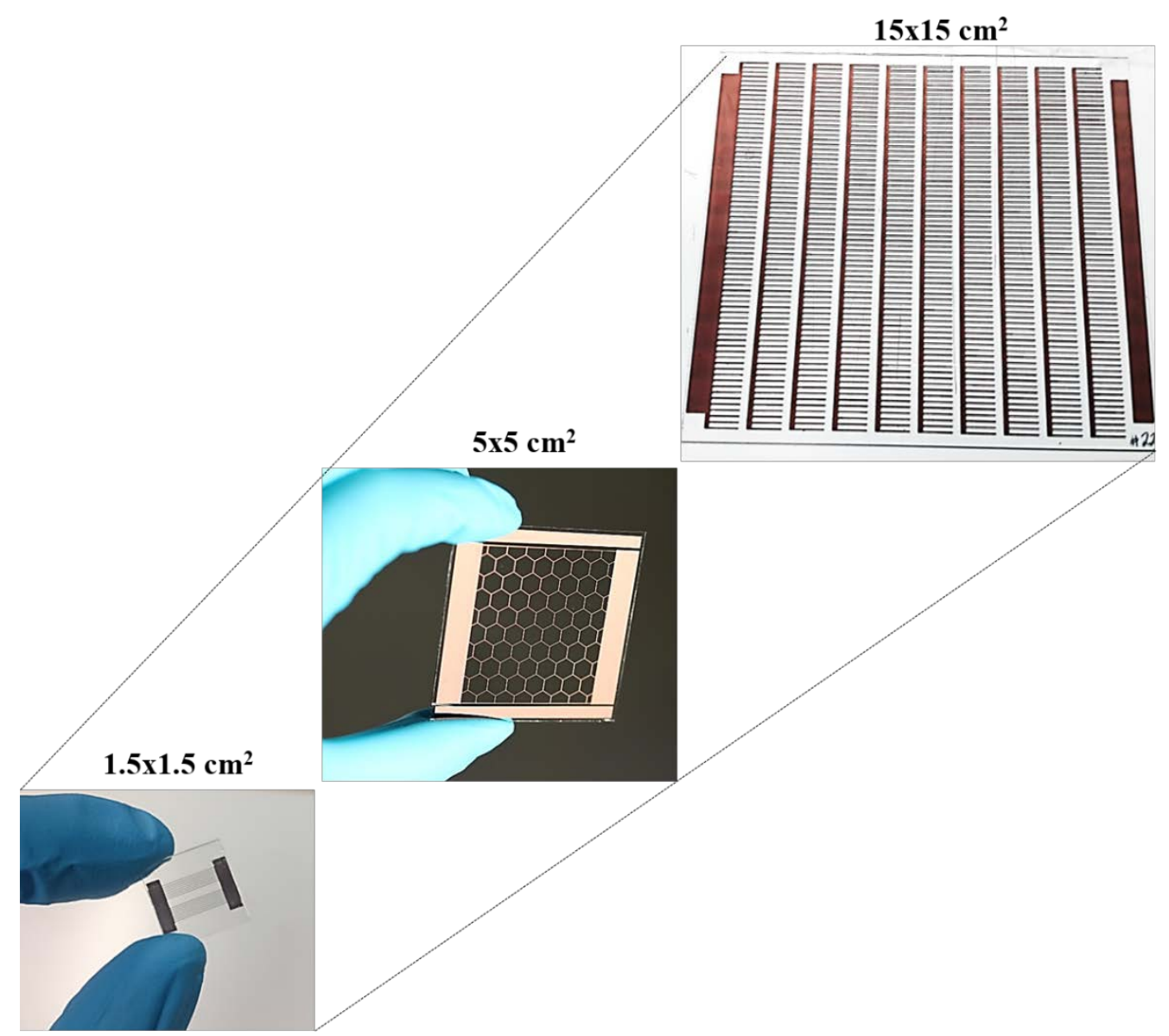

Figure 6: Upscaling of IJP Cu NP-grids on $1.5 \times 1.5 \mathrm{~cm}^{2}, 5 \times 5 \mathrm{~cm}^{2}$ and $15 \times 15 \mathrm{~cm}^{2}$ glass substrates.

One challenging task in parameter optimization for the printing of large area metal grids is their connection to busbars that usually constitutes the simultaneous printing of 1D-structures (grid layout) and 2D structures (busbars). Structures are generally printed with overlapping dots. As a direct consequence, more ink is printed per substrate area for 2D structures, when 1D grids and 2D busbars are printed in the same pattern and when the dpi-value is set to a constant value. In a first approach the printing pattern for honeycomb grids was split into a $1 \mathrm{D}$ and a 2D part. By setting lower dpi values for the busbars compared to the dpi values of the $1 \mathrm{D}$ grid, we could compensate the different amount of printed ink per substrate area. The promising result of the optimized printing and sintering procedure can be seen in Figure 7. The edges of the busbars do not show defects 
and the good connection between grid lines and busbars revealed suitable conductivities for ITO-free Cu-based metal grid OLED processing. Further optimization of the pattern and the printing parameters provided $\mathrm{Cu}$-grids that were printed in a single pattern with a resolution of $600 \mathrm{dpi}$.
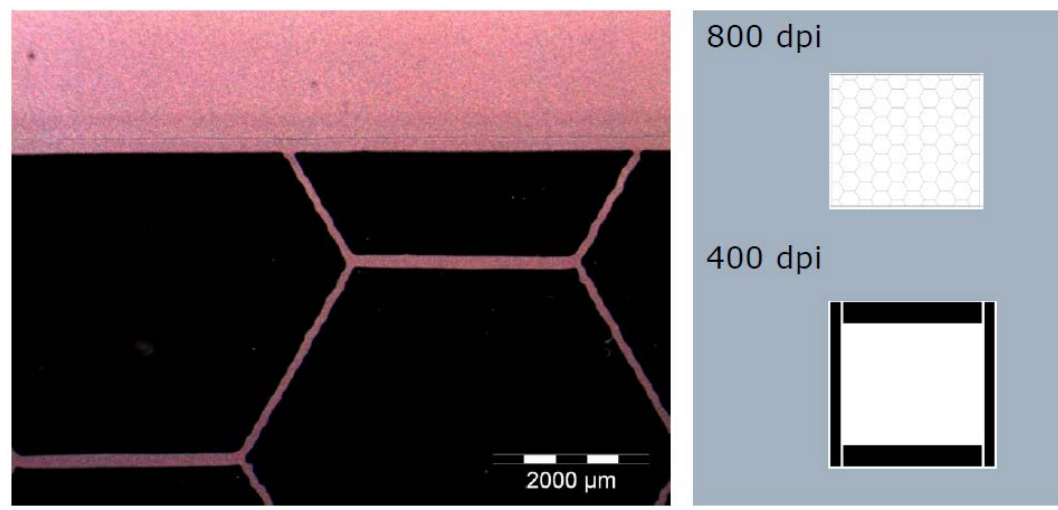

Figure7: Optical image of an optimized honeycomb-busbar structure (left image) and the applied printing patterns with adjusted dpi-resolution (right image).

Laser sintering of grid lines (1D structures), busbars (2D structures) and the connection between lines and areas have different optimized sinter parameters. To avoid over-sintering, the sinter parameters of the entire sample had to be set to the parameters of the most sensitive part, which were the edge of the busbar and the connecting part where the grid line contacts the busbar. Alternatively, it would be possible to sinter different areas of the pattern with different laser parameters, however this more complicated approach sets a high demand on the overlapping of the sintered areas to ensure conductivity.

After the successful printing and sintering of large area grids, reverse nanoimprinting transfer was implemented, in order to flatten the surface of the large area grids. The embedding process required greater care for larger substrates to avoid bubbles in the Ormocomp ${ }^{\circledR}$ resist or breakage of glass substrate, which is more probable with increasing substrate size, however with good care successful embedding could be performed even in $15 \times 15 \mathrm{~cm}^{2}$ glass substrates, as shown in Figure 7. 
(a)

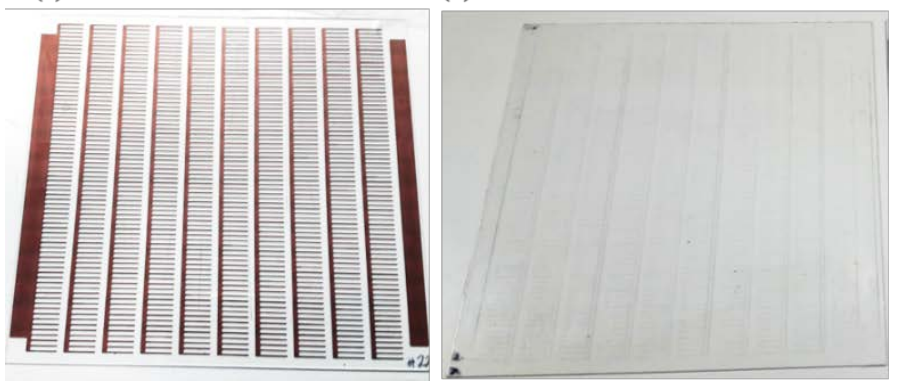

(c)

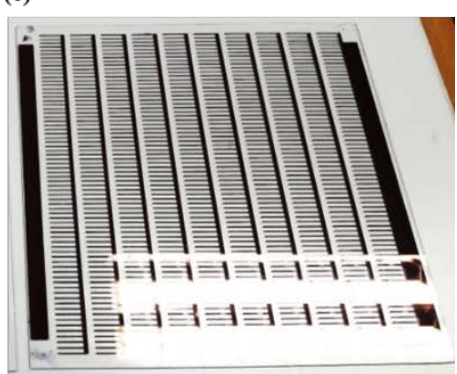

Figure 7: Inkjet-printed Cu NP grid on $15 \times 15 \mathrm{~cm}^{2}$ glass substrate, (a) as printed and sintered on sacrificial glass substrate, (b) sacrificial glass substrate after embedding, and (c) final glass substrate with transferred embedded and flattened Cu NP grid (Please note that the white part within the figure $c$ is not related to grid issues is just an optical effect within the photo due to laboratory light effects)

As a final stage, larger area ITO-free OLED devices were fabricated. The potential of upscaled devices was investigated using $5 \times 5 \mathrm{~cm}^{2}$ glass substrate with embedded Cu NP grid using parallel line layout. As shown in Figure 8, functional upscaled ITO-free OLEDs were fabricated, showing the great potential for future work on large area OLEDs.

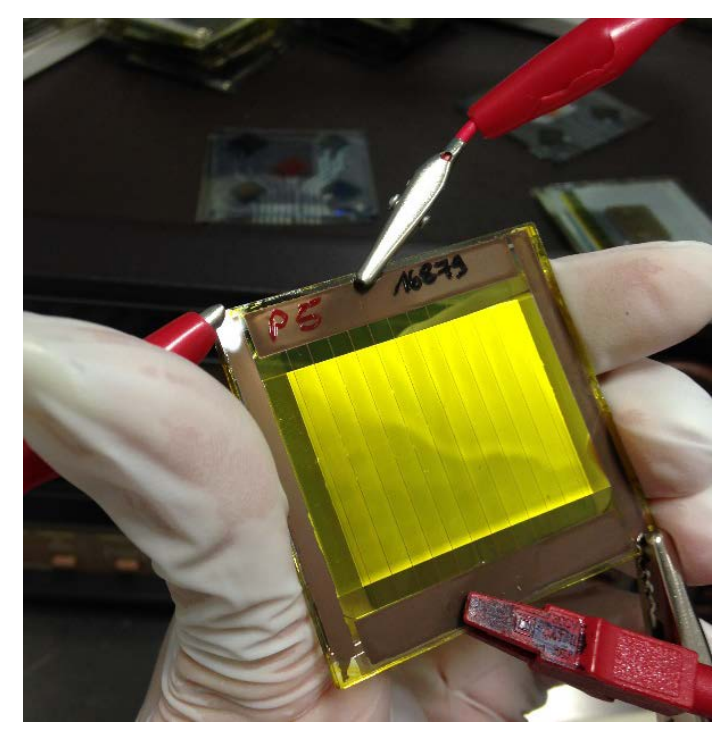

Figure 8: Inkjet-printed OLED on embedded parallel line grid on $5 \times 5 \mathrm{~cm}^{2}$ glass substrates with an active area of $30 \times 40 \mathrm{~mm}^{2}$ 


\section{Conclusion}

We have presented a simple solution-processed OLED device based on an ITOfree inkjet-printed $\mathrm{Cu}$ nanoparticle grid. Starting out from a small active area of $9 \mathrm{~mm}^{2}$, the PEDOT:PSS was optimized for OLED device implementation. A clear trend in efficiency was observed, where current efficiency increased with the conductivity of PEDOT:PSS. Although the ITO-free devices with the highly conductive PEDOT:PSS PH500 showed promising results, a leakage current was present in devices. Studying the leakage losses, it was concluded that the high leakage current of ITO-free devices was a combination of two factors. Leakage that originates from intrinsic properties of high conductive PEDOT:PSS PH500, and leakages from low resistance paths generated in vertical direction between the bottom and top electrodes due to the height of the inkjet-printed Cu NP lines. To reduce the leakage current, embedding $\mathrm{Cu}$ grid methods were implemented by reverse nanoimprinting transfer process to planarize the electrode surface. Important reduction in leakage current and increased current injection efficiency were achieved for embedded $\mathrm{Cu}$ grid-based ITO-free OLEDs. The reported embedded Cu-grid process resulted in $20 \%$ improvement of OLED efficiency in comparison to the performance of ITO-free not embedded grid-based OLEDs. To further optimize the performance of ITO-free OLEDs, alternative grid layouts were compared (lines versus honeycomb designs) and the best performance was achieved using honeycomb grid layout with 23.5\% coverage area. Optimized inkjet-printed Cu-based ITO-free OLEDs reached current efficiency of $3.6 \mathrm{~cd} / \mathrm{A}$, whereas reference ITO-based OLEDs yielded a current efficiency of $5.0 \mathrm{~cd} / \mathrm{A}$. Additionally, we prove that the whole printing, sintering and embedding process of the developed Cu-grids can be applied for the fabrication of flexible and large area OLEDs indicating the prospect of the developed printed $\mathrm{Cu}$-based electrode for truly low-cost OLED fabrication. 


\section{Experimental}

Lab-scale $1.5 \times 1.5 \mathrm{~cm}$ soda-lime glass substrates were sonicated in acetone and subsequently in isopropanol for $10 \mathrm{~min}$ followed by additional $10 \mathrm{~min}$ of $\mathrm{UV}-\mathrm{O}_{3}$ treatment before printing of metal grids. For the $5 \times 5 \mathrm{~cm}^{2}$ and $15 \times 15 \mathrm{~cm}^{2}$ substrates Corning EAGLE XG glass was used prior cleaning in a hot Hellmanexsolution, followed by a thorough rinsing with water.

The CI-005 Cu-NP ink (Intrinsiq Materials) was produced using copper nanoparticles of approximately $100 \mathrm{~nm}$ in diameter, which had been formed by a high temperature plasma synthesis method. The nanoparticles were added to a blend of solvents containing a small quantity of additives and resin and dispersed using a bead mill. The dispersion was then filtered and adjusted to a viscosity of approximately $12 \mathrm{cP}$ with additional solvent. The surface tension of the ink was $29-30 \mathrm{mN} \mathrm{m}^{-1}$.

This copper nanoparticle-based ink was inkjet-printed using a Fujifilm Dimatix DMP-2800 piezoelectric drop on demand printer with a $10 \mathrm{pL}$ cartridge for the case of lab-scale samples, on the other hand PixDro LP50 printer with Spectra S class SE128 printhead with a drop volume of 30 pL was used for the printing of upscaled grids. All the printing processes were performed under ambient conditions. The substrate and cartridge temperatures were set at $35^{\circ} \mathrm{C}$. The print head was set at $700 \mu \mathrm{m}$ above the substrate and $\mathrm{Cu}$ structures were printed with $25 \mu \mathrm{m}$ drop spacing resulting in lines of $405 \pm 51 \mathrm{~nm}$ average height and 80-85 $\mu \mathrm{m}$ width as measured with a Veeco Dektak 150 profilometer.

The IJP Cu grids were dried on a hotplate at $60{ }^{\circ} \mathrm{C}$ for $1 \mathrm{~h}$. An infra-red diode laser (LAPS 60, Intrinsiq Materials) with an output current of 55 A, $808 \mathrm{~nm}$ beam, $25 \mathrm{~mm} \cdot \mathrm{s}^{-1}$ speed and $14 \mathrm{~mm}$ between a focusing lens and the sample surface was used to sinter the IJP Cu grids.

Various PEDOT:PSS formulations were purchased from Heraeus (Clevios ${ }^{\mathrm{TM}} \mathrm{P}$ VP AI 4083, Clevios ${ }^{\mathrm{TM}}$ F CE and Clevios ${ }^{\mathrm{TM}} \mathrm{PH}$ 500) and investigated in this study. For non-embedded IJP Cu grids, 100-120 nm PEDOT:PSS were spin coated to overcoat the $\mathrm{Cu}$ lines while 40-45 nm thickness was used on-top of embedded grids. Annealing of PEDOT:PSS layers was performed at $140{ }^{\circ} \mathrm{C}$ on a hotplate for $20 \mathrm{~min}$ in ambient conditions. For the embedding of IJP Cu grid, we 
followed the reverse nanoimprinting transfer procedure ${ }^{15}$, where Ormocomp@ and Ormoprime $@$ resins were purchased from Micro Resist Technology. For the embedding process using a flexible substrate (Melinex 506) we used the internally developed resist NILcure JR82. Pre-patterned ITO substrates (sheet resistance $4 \Omega \mathrm{sq}^{-1}$ ) were purchased from Psiotec Ltd. The OLED active layer material PDY-132 (Super Yellow) was purchased from Merck and dissolved in toluene with a concentration of $6.5 \mathrm{mg} / \mathrm{ml}$. The solution was spin coated on PEDOT:PSS resulting in a layer thickness of $75-80 \mathrm{~nm}$ and annealed at $50{ }^{\circ} \mathrm{C}$ for 20 minutes. Both spin coating and annealing were performed, inside nitrogen atmosphere glovebox. To complete the normal-structured stack, $1 \mathrm{~nm}$ of lithium fluoride and $100 \mathrm{~nm}$ of aluminum both of which were thermally evaporated on top of the photoactive layer and patterned through a shadow mask, resulting in four light emitting diodes, each with an active area of $9 \mathrm{~mm}^{2}$. Topographic analyses were performed using a Zeiss Gemini SEM. A four-point probe (Jandel RM3000) conductivity meter was employed for sheet resistance measurements. The LIV characteristics were acquired using a Botest measurement setup. Photocurrent mapping measurements were performed under 405nm laser excitation using a Botest PCT photocurrent system.

\section{Acknowledgment}

We acknowledge funding from the PLASMAS project through the European Union (FP7 Project No. 604568). The ink development was performed while Intrinsiq Materials Farnborough was still under operation before their closing in August 2018 by Richard Ward (current address: rward6@sky.com) and Charlotte Rapley. 


\section{References}

1. Goonan, T. G. Materials Flow of Indium in the United States in 2008 and 2009. 20 (2012).

2. Mazzeo, M., Mariano, F., Genco, A., Carallo, S. \& Gigli, G. High efficiency ITO-free flexible white organic light-emitting diodes based on multi-cavity technology. Org. Electron. physics, Mater. Appl. 14, 28402846 (2013).

3. Ouyang, S. et al. P-148: Highly Conductive PEDOT:PSS Patterning Scheme and its Application to ITO-free Organic Light Emitting Diodes. SID Symp. Dig. Tech. Pap. 45, 1540-1543 (2014).

4. Yousefi, M. H., Fallahzadeh, A., Saghaei, J. \& Darareh, M. D. Fabrication of flexible ITO-Free OLED using vapor-treated PEDOT:PSS thin film as anode. J. Disp. Technol. 12, 1647-1651 (2016).

5. Morales-Masis, M. et al. An Indium-Free Anode for Large-Area Flexible OLEDs: Defect-Free Transparent Conductive Zinc Tin Oxide. Adv. Funct. Mater. 26, 384-392 (2016).

6. Hu, L., Li, J., Liu, J., Gr??ner, G. \& Marks, T. Flexible organic lightemitting diodes with transparent carbon nanotube electrodes: Problems and solutions. Nanotechnology 21, (2010).

7. Reboud, V. et al. Enhanced light extraction in ITO-free OLEDs using double-sided printed electrodes. Nanoscale 4, 3495 (2012).

8. Bocksrocker, T. et al. Highly efficient fully flexible indium tin oxide free organic light emitting diodes fabricated directly on barrier-foil. Thin Solid Films 542, 306-309 (2013).

9. Zhou, L. et al. High-Performance Flexible Organic Light-Emitting Diodes Using Embedded Silver Network Transparent Electrodes. ACS Nano 8, 12796-12805 (2014).

10. Sam, F. L. M. et al. Hybrid metal grid-polymer-carbon nanotube electrodes for high luminance organic light emitting diodes. Nanotechnology 25, (2014). 
11. Alshammari, A. S. et al. Controlled growth and spray deposition of silver nanowires for ITO-free, flexible, and high brightness OLEDs. Phys. Status Solidi Appl. Mater. Sci. 214, 1-10 (2017).

12. Galagan, Y. et al. Current collecting grids for ITO-free solar cells. Adv. Energy Mater. 2, 103-110 (2012).

13. Galagan, Y. et al. ITO-free flexible organic solar cells with printed current collecting grids. Sol. Energy Mater. Sol. Cells 95, 1339-1343 (2011).

14. Neophytou, M., Hermerschmidt, F., Savva, A., Georgiou, E. \& Choulis, S. A. Highly efficient indium tin oxide-free organic photovoltaics using inkjet-printed silver nanoparticle current collecting grids. Appl. Phys. Lett. 101, 193302 (2012).

15. Burgués-Ceballos, I., Kehagias, N., Sotomayor-Torres, C. M., CampoyQuiles, M. \& Lacharmoise, P. D. Embedded inkjet printed silver grids for ITO-free organic solar cells with high fill factor. Sol. Energy Mater. Sol. Cells 127, 50-57 (2014).

16. Georgiou, E. et al. Printed Copper Nanoparticle Metal Grids for CostEffective ITO-Free Solution Processed Solar Cells. Sol. RRL 1700192, 1700192 (2018).

17. Galagan, Y. et al. Solar Energy Materials \& Solar Cells Evaluation of inkjet printed current collecting grids and busbars for ITO-free organic solar cells. Sol. Energy Mater. Sol. Cells 104, 32-38 (2012).

18. Hermerschmidt, F. et al. High performance indium tin oxide-free solutionprocessed organic light emitting diodes based on inkjet-printed fine silver grid lines. Flex. Print. Electron. 1, 035004 (2016).

19. Kinner, L. et al. Inkjet-printed embedded Ag-PEDOT:PSS electrodes with improved light out coupling effects for highly efficient ITO-free blue polymer light emitting diodes. Appl. Phys. Lett. 110,101107 (2017).

20. Harkema, S. et al. Large area ITO-free flexible white OLEDs with Orgacon PEDOT:PSS and printed metal shunting lines. 7415, 74150T (2009).

21. ICSG. The World Copper Factbook. (2014). 
22. Park, B. K., Kim, D., Jeong, S., Moon, J. \& Kim, J. S. Direct writing of copper conductive patterns by ink-jet printing. Thin Solid Films 515, 7706-7711 (2007).

23. Kang, J. S. et al. Inkjet printed electronics using copper nanoparticle ink. J. Mater. Sci. Mater. Electron. 21, 1213-1220 (2010).

24. Jang, S. et al. Sintering of inkjet printed copper nanoparticles for flexible electronics. Scr. Mater. 62, 258-261 (2010).

25. Lee, Y., Choi, J. R., Lee, K. J., Stott, N. E. \& Kim, D. Large-scale synthesis of copper nanoparticles by chemically controlled reduction for applications of inkjet-printed electronics. Nanotechnology 19, (2008).

26. Farraj, Y., Grouchko, M. \& Magdassi, S. Self-reduction of a copper complex MOD ink for inkjet printing conductive patterns on plastics. Chem. Commun. 51, 1587-1590 (2015).

27. Norita, S. et al. Inkjet-printed copper electrodes using photonic sintering and their application to organic thin-film transistors. Org. Electron. physics, Mater. Appl. 25, 131-134 (2015).

28. Polino, G. et al. A benchmark study of commercially available copper nanoparticle inks for application in organic electronic devices. Org. Electron. physics, Mater. Appl. 34, 130-138 (2016).

29. Hermerschmidt, F. et al. Truly Low Temperature Sintering of Printed Copper Ink Using Formic Acid. Adv. Mater. Technol. 1800146, 1800146 (2018).

30. Niittynen, J., Sowade, E., Kang, H., Baumann, R. R. \& Mäntysalo, M. Comparison of laser and intense pulsed light sintering (IPL) for inkjetprinted copper nanoparticle layers. Sci. Rep. 5, 8832 (2015).

31. Soltani, A., Khorramdel Vahed, B., Mardoukhi, A. \& Mäntysalo, M. Laser sintering of copper nanoparticles on top of silicon substrates. Nanotechnology 27, (2015).

32. Zenou, M., Ermak, O., Saar, A. \& Kotler, Z. Laser sintering of copper nanoparticles. J. Phys. D. Appl. Phys. 47, (2014). 
33. Hwang, H. J., Oh, K. H. \& Kim, H. S. All-photonic drying and sintering process via flash white light combined with deep-UV and near-infrared irradiation for highly conductive copper nano-ink. Sci. Rep. 6, 1-10 (2016).

34. Division, E. M. Clevios ${ }^{\mathrm{TM}}$ f ce. 5-6 (2014).

35. Mauger, S. A., Chang, L., Rochester, C. W. \& Moulé, A. J. Directional dependence of electron blocking in PEDOT:PSS. Org. Electron. 13, 27472756 (2012).

36. Savva, A. et al. Photovoltaic analysis of the effects of PEDOT:PSSadditives hole selective contacts on the efficiency and lifetime performance of inverted organic solar cells. Sol. Energy Mater. Sol. Cells 132, 507-514 (2015).

37. Shi, H., Liu, C., Jiang, Q. \& Xu, J. Effective Approaches to Improve the Electrical Conductivity of PEDOT:PSS: A Review. Adv. Electron. Mater. 1, 1-16 (2015). 


\section{Supporting Information}

\section{Optimization of grid coverage area}

Grid coverage area has been already investigated in the literature, and it had been proven that the optimum coverage is strongly depended on the materials of the composite electrode ${ }^{12,16-18}$. Since the current work investigates a totally new hybrid electrode where $\mathrm{Cu} \mathrm{NP}$ grid is combined with highly conductive PEDOT:PSS PH500 and used in OLED devices, optimum grid coverage area was necessary to be investigated. Parallel line layout was inkjet-printed with 4, 6, 8, and 10 lines, to include and investigate grid coverage range of $10-30 \%$. All the grids were embedded and then spin coated with a thin (40-45nm) layer of highly conductive PEDOT:PSS PH500.

Table S1: Statistical analysis of electroluminescence performance of lab-scale $9 \mathbf{~ m m}^{2}$ ITOfree OLED devices, using embedded parallel grids with various covered areas. Statistic sample size of 8 different devices was used to present average values, together with standard deviation, and maximum values in brackets.

\begin{tabular}{|c|c|c|c|c|c|}
\hline Device structure & $\begin{array}{c}\text { Grid } \\
\text { covered } \\
\text { area }\end{array}$ & $\begin{array}{c}\text { Turn on } \\
\text { voltage } \\
\left.\text { (at } 10 \mathrm{~cd} / \mathbf{m}^{2}\right) \\
{[\mathrm{V}]}\end{array}$ & $\begin{array}{c}\text { Max. } \\
\text { Luminance } \\
{\left[\mathbf{c d} / \mathbf{m}^{2}\right]}\end{array}$ & $\begin{array}{c}\text { Max. Current } \\
\text { efficiency } \\
\text { [cd/A] }\end{array}$ & $\begin{array}{c}\text { Max. Power } \\
\text { Efficiency } \\
\text { [lm/W] }\end{array}$ \\
\hline Reference & --- & 2.6 & $9.0 \mathrm{k} \pm 0.3 \mathrm{k}$ & $4.7 \pm 0.2(4.9)$ & $4.0 \pm 0.5(4.5)$ \\
\hline ITO-free; 4 lines & $11.7 \%$ & 3.3 & $4.0 \mathrm{k} \pm 4.0 \mathrm{k}$ & $1.7 \pm 0.6(2.2)$ & $0.8 \pm 0.4(1.1)$ \\
\hline ITO-free; 6 lines & $17.6 \%$ & 2.9 & $8.0 \mathrm{k} \pm 2.0 \mathrm{k}$ & $2.3 \pm 0.4(2.8)$ & $1.4 \pm 0.7(2.4)$ \\
\hline ITO-free; 8 lines & $23.5 \%$ & 2.9 & $9.0 \mathrm{k} \pm 0.6 \mathrm{k}$ & $2.3 \pm 0.5(3.0)$ & $1.5 \pm 0.8(2.5)$ \\
\hline ITO-free; 10 lines & $29.3 \%$ & 3.2 & $6.0 \mathrm{k} \pm 2.0 \mathrm{k}$ & $1.3 \pm 0.1(1.3)$ & $0.7 \pm 0.4(1.3)$ \\
\hline \multicolumn{4}{|c|}{$\begin{array}{l}\text { Reference: ITO/AI4083/SY/LiF/Al } \\
\text { ITO-free: Embedded parallel line Cu grid/PH500/ SY/LiF/Al }\end{array}$} & \multicolumn{2}{|c|}{$\frac{\text { Lines } \mathrm{x} \text { (Width } \mathrm{x} \text { Length) }}{\text { Total device area }}$} \\
\hline
\end{tabular}




\section{Importance of grid presence in optimized lab scale ITO-free OLEDs}

To support the high efficiency of the optimized ITO-free OLED devices, the importance of $\mathrm{Cu}$ grid presence was investigated by comparing them with devices using only high conductive PEDOT: PSS PH 500. Both ITO-free devices consisted of identical HIL, LEP and top electrode, therefore the only difference was the presence of Cu grid. Observing the JV characteristics in Figure S 2a, PH 500- 'only' devices showed very low charge injection in forward bias in comparison to the devices with Cu-based electrodes. The problem in charge injection of PH 500- 'only' devices was also confirmed by the high turn on voltage, as shown in Figure S 2b. Inefficient charge injection had a significant impact on the maximum luminance and power efficiency of the ITO-free devices, as shown in Figure S 2c. Therefore, even in small area devices the importance of grid presence was underlined. The presence of metal grid significantly improves the electrical properties of the devices and increases the overall performance of ITO-free OLEDs.
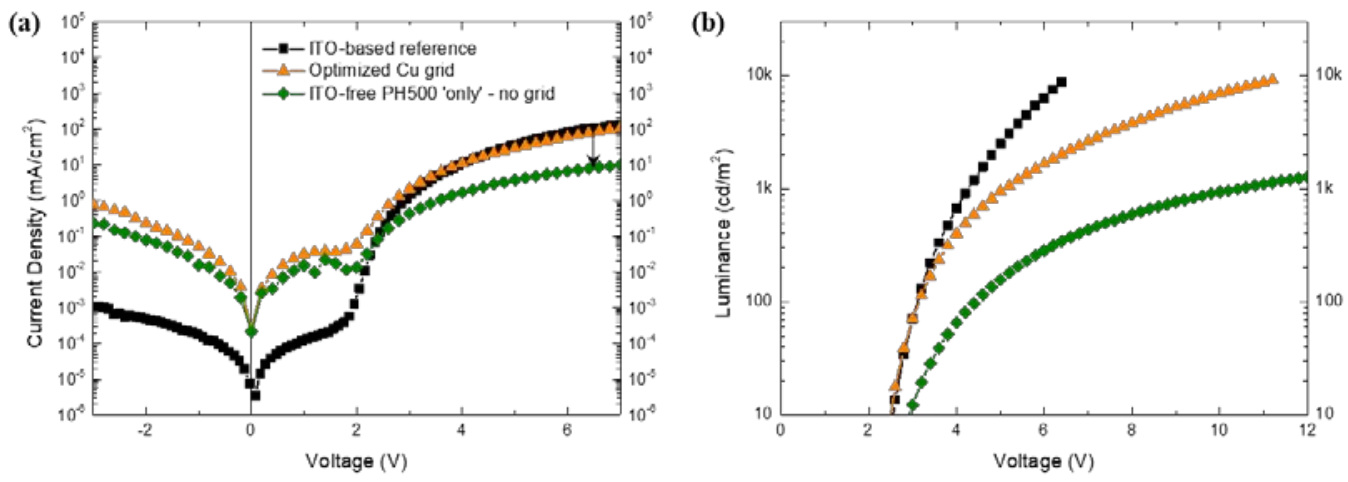

Figure S 1: (a) Linear JV plot, (b) luminance characteristics and (c) power efficiency of labscale 9 mm² ITO-free OLEDs; embedded honeycomb Cu grid vs PH500-‘only' bottom electrodes. 


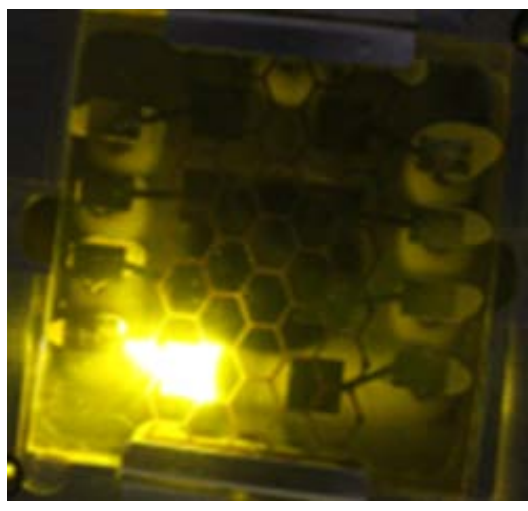

Figure S 2: Lab-scale $9 \mathbf{~ m m}^{2}$ flexible ITO-free OLED device under operation, using embedded honeycomb Cu grid/PEDOT:PSS hybrid bottom electrode. 